TRANSACTIONS OF THE

AMERICAN MATHEMATICAL SOCIETY

Volume 349, Number 4, April 1997, Pages 1653-1662

S 0002-9947(97)01894-1

\title{
DE RHAM COHOMOLOGY OF LOGARITHMIC FORMS ON ARRANGEMENTS OF HYPERPLANES
}

\author{
JONATHAN WIENS AND SERGEY YUZVINSKY
}

\begin{abstract}
The paper is devoted to computation of the cohomology of the complex of logarithmic differential forms with coefficients in rational functions whose poles are located on the union of several hyperplanes of a linear space over a field of characteristic zero. The main result asserts that for a vast class of hyperplane arrangements, including all free and generic arrangements, the cohomology algebra coincides with the Orlik-Solomon algebra. Over the field of complex numbers, this means that the cohomologies coincide with the cohomologies of the complement of the union of the hyperplanes. We also prove that the cohomologies do not change if poles of arbitrary multiplicity are allowed on some of the hyperplanes. In particular, this gives an analogue of the algebraic de Rham theorem for an arbitrary arrangement over an arbitrary field of zero characteristic.
\end{abstract}

\section{INTRODUCTION}

In topological theory of hyperplane arrangements the most often cited result is the Brieskorn theorem [1]. Let $\mathcal{A}$ be an arrangement of hyperplanes in a linear space $V$ over the complex field $\mathbb{C}$ defined by the polynomial $Q=\prod_{H \in \mathcal{A}} \alpha_{H}$ where $\alpha_{H} \in V^{*}$ and $\operatorname{ker} \alpha_{H}=H$. Let $M=V \backslash \bigcup_{H \in \mathcal{A}} H$. Then the theorem says that the algebra $H^{*}(M ; \mathbb{C})$ is isomorphic under the de Rham homomorphism to the graded $\mathbb{C}$-algebra $A=A(\mathcal{A})$ of differential forms generated by the forms $d \alpha_{H} / \alpha_{H}(H \in \mathcal{A})$. On the other hand, the most popular object of algebraic theory of arrangements is the algebra $\Omega=\Omega(\mathcal{A})$ (over a polynomial ring) of logarithmic differential forms on $\mathcal{A}$. This algebra has been introduced by K. Saito and consists of all rational forms $\omega$ on $V$ such that both $Q \omega$ and $Q d \omega$ are regular. Algebra $\Omega(\mathcal{A})$, graded by the order of the forms and provided with the exterior differential, forms a complex $\Omega^{*}=\Omega^{*}(\mathcal{A})$ and it is natural to try to compute its cohomology. Notice that $A(\mathcal{A}) \subset \Omega^{*}(\mathcal{A})$ as a subcomplex with 0 differential. Computing simple examples prompts the conjecture that over any field of 0 characteristic the embedding $A(\mathcal{A}) \subset \Omega^{*}(\mathcal{A})$ is a quasi isomorphism, in particular $H^{*}\left(\Omega^{*}\right)=A$. Over $\mathbb{C}$ a partial support for this conjecture is given by the Algebraic de Rham Theorem by Grothendieck [4] that asserts that the cohomology of the larger complex $\Omega^{*}(* \mathcal{A})$ of forms with poles of arbitrary multiplicity on the hyperplanes is $H^{*}(M ; \mathbb{C})$ that is again $A(\mathcal{A})$ by the Brieskorn theorem.

This conjecture first appeared in the literature in a slightly hidden form in [11] where it is the specification of Conjecture 3.1 for the arrangement case. It was proved there for the first cohomology (in a more general context). G.Ziegler made

Received by the editors November 15, 1994.

1991 Mathematics Subject Classification. Primary 52B30, 14F40, 05B35.

(C)1997 American Mathematical Society 
an attempt to prove the conjecture in full generality in his $\mathrm{Ph}$. D. Thesis at MIT in 1987 but H.Terao found an error in his proof. Recently F.Castro, L.Narváez, and D.Mond [2] proved the analogue of the conjecture in analytic category (i.e., for meromorphic forms) for free arrangements, in fact for every strongly quasihomogeneous free divisor. H.Terao observed that their method works for tame arrangements also (in analytic category). Using this method and the results for small dimensions, he and the second author covered the case in analytic category of arbitrary arrangements (over $\mathbb{C}$ ) in dimension less than 5 .

In this paper we prove the conjecture over an arbitrary field of characteristic 0 for the class of tame arrangements that includes generic arrangements, free arrangements, and all arrangements in dimension less than 4 . In fact we prove a more general result by considering forms with mixed conditions on the order of their poles. In particular we obtain a direct algebraic proof that $H^{*}\left(\Omega^{*}(* \mathcal{A})\right)=A(\mathcal{A})$. We also prove that conjecture is true for the second and the highest cohomology for an arbitrary arrangement whence for all arrangements in dimension 4 .

The second author would like to thank H.Terao for useful discussions of the subject and D.Mond and L.Narváez for explaining to him the subtle points of their proof.

\section{Setup AND MAIN THEOREM}

Throughout $F$ will be a field of characteristic 0 and $V$ an $\ell$-dimensional linear space over $F$. Let $\mathcal{A}$ be a central arrangement of (linear) hyperplanes in $V$ defined by a polynomial $Q=Q(\mathcal{A})=\prod_{H \in \mathcal{A}} \alpha_{H}$ where $\alpha_{H} \in V^{*}$ and ker $\alpha_{H}=H$. Every subarrangement $\mathcal{B}$ of $\mathcal{A}$ can be defined by the polynomial $Q(\mathcal{B})=\prod_{H \in \mathcal{B}} \alpha_{H}$. The intersection lattice $L=L(\mathcal{A})$ of $\mathcal{A}$ is (in this paper) the set of all intersections of elements from $\mathcal{A}$ ordered by inclusion. In particular the maximal element of $L$ is $V$ and its minimal element is $U=\bigcap_{H \in \mathcal{A}} H$. If $U=0$ the arrangement is called essential. For any arrangement $\mathcal{A}$ the functionals $\alpha_{H}$ define the essential arrangement $\mathcal{A}_{e}$ in $V / U$. The lattice $L$ is ranked and we put $\operatorname{rk} \mathcal{A}=\operatorname{rk} L$. Clearly $\operatorname{rk} \mathcal{A}+\operatorname{dim} U=\ell$. Every $X \in L$ defines the subarrangement $\mathcal{A}_{X}=\{H \in \mathcal{A} \mid X \subset H\}$ of $\mathcal{A}$ of rank $\ell-\operatorname{dim}_{F} X$. These subarrangements can be characterized among all subarrangements of $\mathcal{A}$ as being linearly closed in $\mathcal{A}$. This means that for every $H \in \mathcal{A}$ such that $\alpha_{H}$ is a linear combination of $\alpha_{K}$ with $K \in \mathcal{A}_{X}$ we have $H \in \mathcal{A}_{X}$.

We consider several graded algebras over the symmetric algebra $S\left(V^{*}\right)$ of $V^{*}$. For any basis $\left(x_{1}, \ldots, x_{\ell}\right)$ of $V^{*}$ we will identify $S\left(V^{*}\right)$ with the polynomial algebra $S=F\left[x_{1}, \ldots, x_{\ell}\right]$. All $S$-algebras consist of differential forms on $V$ with rational cofficients. The largest algebra $\Omega(* \mathcal{A})$ is the algebra of all the forms whose denominators are products of $\alpha_{H}(H \in \mathcal{A})$ with arbitrary multiplicities. Then we fix a pair of arrangements $(\mathcal{A}, \mathcal{B})(\mathcal{B} \subset \mathcal{A})$ and put

$$
\Omega(\mathcal{A}, \mathcal{B})=\{\omega \in \Omega(* \mathcal{A}) \mid Q(\mathcal{B}) \omega, Q(\mathcal{B}) d \omega \in \Omega(*(\mathcal{A} \backslash \mathcal{B}))\} .
$$

Notice that $\Omega(\mathcal{A}, \emptyset)=\Omega(* \mathcal{A})$ and $\Omega(\mathcal{A}, \mathcal{A})=\Omega(\mathcal{A})$, that is, the algebra of all logarithmic forms on $\mathcal{A}$ (see Introduction). Also notice that

$$
\Omega(\mathcal{A}, \mathcal{B})=\Omega(\mathcal{A})_{Q / Q(\mathcal{B})}=\Omega(\mathcal{B})_{Q / Q(\mathcal{B})}
$$

where a polynomial as a subindex means the localization at the multiplicative set generated by the irreducible factors of this polynomial.

Algebra $\Omega(\mathcal{A}, \mathcal{B})$ is graded by the order of the forms and the exterior differential $d$ makes it into a cochain complex. We denote this complex by $\Omega^{*}(\mathcal{A}, \mathcal{B})$ omitting 
$\mathcal{A}$ and $\mathcal{B}$ when they are fixed. Explicitly the complex is

$$
0 \rightarrow \Omega^{0} \stackrel{d}{\rightarrow} \Omega^{1} \stackrel{d}{\rightarrow} \cdots \stackrel{d}{\rightarrow} \Omega^{\ell} \rightarrow 0 .
$$

Any pair $(\mathcal{A}, \mathcal{B})$ can be reduced to the pair of arrangements in $V / U$ (defined by the same functionals) with $\mathcal{A}_{e}$ in place of $\mathcal{A}$. Although the complex $\Omega^{*}(\mathcal{A}, \mathcal{B})$ becomes in general smaller under this reduction its cohomology does not change. Thus we will often assume that $\mathcal{A}$ is essential.

In order to formulate the main theorem we need to introduce an important class of arrangements.

Definition 2.1. A central arrangement $\mathcal{A}$ is called tame if for every $p$ one has

$$
\operatorname{pd}_{S} \Omega^{p}(\mathcal{A}) \leq p
$$

where $\operatorname{pd}_{S}$ means the projective dimension of an $S$-module.

This property has been used first in [8], the terminology appeared in [13]. Clearly any free arrangement is tame. Due to [9] every generic arrangement is tame also. It is not hard to verify directly that every 3 -arrangement is tame. The smallest known example of a non-tame arrangement is contained in [8]. It is the arrangement given in $\left\{\left(x_{1}, x_{2}, x_{3}, x_{4}\right)\right\}$ by the 15 non-zero linear functionals $a_{1} x_{1}+a_{2} x_{2}+a_{3} x_{3}+a_{4} x_{4}$ with $a_{i}=0,1$.

Let us observe properties of tame arrangements we will need to use in this paper.

(T1) Let $\mathcal{A}$ be an arbitrary arrangement. For each subspace $U_{0} \subset U$ the functionals of hyperplanes from $\mathcal{A}$ define an arrangement in the space $V / U_{0}$. If $\mathcal{A}$ is tame then all these arrangements are tame also, in particular $\mathcal{A}_{e}$ is tame.

Proof. We consider the case $U_{0}=U$ only. Suppose $\mathcal{A}$ is tame and fix $p, 0 \leq p \leq$ $\operatorname{rk} \mathcal{A}$. It is well known (cf. [10], Proposition 5.8) that

$$
\Omega^{p}(\mathcal{A}) \cong \bigoplus_{q=0}^{p} \Omega^{q}\left(\mathcal{A}_{e}\right) \otimes_{F} \Omega^{p-q}(\Phi)
$$

where $\Phi$ is the empty arrangement in $U$. Here $\Omega^{q}\left(\mathcal{A}_{e}\right)$ is a module over $S_{0}=$ $S\left((V / U)^{*}\right), \Omega^{p-q}(\Phi)$ is a free module over $S_{1}=S\left(U^{*}\right)$, and the tensor product defines $\Omega^{p}(\mathcal{A})$ as the module over $S \cong S_{0} \otimes_{F} S_{1}$. For each $q, 0 \leq q \leq p$, fix a minimal graded free resolution $R_{q}$ of the graded $S_{0}$-module $\Omega^{q}\left(\mathcal{A}_{e}\right)$. Then $R=$ $\bigoplus_{q=0}^{p} R_{q} \otimes_{F} \Omega^{p-q}(\Phi)$ is a graded free resolution of the graded $S$-module $\Omega^{p}(\mathcal{A})$. Using the graded version of the criterion from [12], p. 54, Lemma 4.4, it is easy to see that $R$ is minimal. Thus the length of $R$ is less than or equal to $p$ whence the same is true for the length of $R_{p}$. That completes the proof.

(T2) If $\mathcal{A}$ is tame then $\mathcal{A}_{X}$ is tame for every $X \in L$.

Proof. It suffices to prove that $\operatorname{pd}_{S} \Omega^{p}\left(\mathcal{A}_{X}\right) \leq \operatorname{pd}_{S} \Omega^{p}(\mathcal{A})$. One can prove this following the proof of [14], Lemma 2.1, and using that $\Omega^{p}\left(\mathcal{A}_{X}\right)_{\mathcal{M}} \cong \Omega^{p}(\mathcal{A})_{\mathcal{M}}$ where $\mathcal{M}$ is the maximal ideal of $S$ corresponding to any generic point from $X$.

Now we formulate the main results of the paper.

Theorem 2.2. Suppose that $\mathcal{A}_{X}$ is tame whenever $\mathcal{A}_{X} \subset \mathcal{B}$. Then the natural embedding

$$
A(\mathcal{A}) \subset \Omega^{*}(\mathcal{A}, \mathcal{B})
$$

is a quasi-isomorphism. 
Corollary 2.3. For every tame arrangement $\mathcal{A}$ the natural embedding

$$
A(\mathcal{A}) \subset \Omega^{*}(\mathcal{A})
$$

is a quasi-isomorphism.

This is the particular case of Theorem 2.2 for $\mathcal{B}=\mathcal{A}$ taking (T2) into consideration.

Corollary 2.4. For every tame arrangement $\mathcal{A}$ over the complex field $\mathbb{C}$ and every $p$ we have

$$
H^{p}\left(\Omega^{*}(\mathcal{A})\right) \cong H^{p}(M ; \mathbb{C})
$$

and the isomorphism is induced by the de Rham map.

This follows from Corollary 2.3 and the Brieskorn theorem [1].

Corollary 2.5. For every arrangement $\mathcal{A}$ the natural embedding

$$
A(\mathcal{A}) \subset \Omega^{*}(* \mathcal{A})
$$

is a quasi-isomorphism.

This is the particular case of Theorem 2.2 for $\mathcal{B}=\emptyset$.

The next two sections of the paper are devoted to the proof of Theorem 2.2.

\section{Cohomology of Sheaves on $L$}

In this section we represent $\Omega^{*}(\mathcal{A}, \mathcal{B})$ as the complex of global sections of certain sheaves on the poset $L_{0}=L \backslash\{U\}$ where $L$ is the intersection lattice of $\mathcal{A}$ and prove that low cohomlogies of these sheaves vanish.

Fix $q$ and for every $X \in L_{0}$ put $\mathcal{F}^{q}(X)=\Omega^{q}\left(\mathcal{A}, \mathcal{B} \cap \mathcal{A}_{X}\right)$. If $X \leq Y$ in $L_{0}$ then $\mathcal{F}^{q}(X) \subset \mathcal{F}^{q}(Y)$. Using these embeddings as structure homomorphisms we obtain a presheaf whose sheafification is the sheaf $\mathcal{F}^{q}$ on $L_{0}$ where $L_{0}$ is provided with the usual topology of all the increasing subsets.

Lemma 3.1. If $\operatorname{rk} \mathcal{A}>1$ then $\mathcal{F}^{q}\left(L_{0}\right)=\Omega^{q}(\mathcal{A}, \mathcal{B})$.

Proof. The natural embeddings $\Omega^{q}(\mathcal{A}, \mathcal{B}) \subset \Omega^{q}\left(\mathcal{A}, \mathcal{B} \cap \mathcal{A}_{X}\right)\left(X \in L_{0}\right)$ define an embedding $\Omega^{q}(\mathcal{A}, \mathcal{B}) \subset \mathcal{F}^{q}\left(L_{0}\right)$. We need to prove only that it is surjective.

Let $s \in \mathcal{F}^{q}\left(L_{0}\right)$. Since $L_{0}$ has the unique maximal element $V$ and all structure homomorphisms of our sheaves are injective the form $\omega=s(X)$ does not depend on $X$. Since rk $L>1$ we have $H \in L_{0}$ for every $H \in \mathcal{A}$. If $H \in \mathcal{B}$ then we have $\alpha_{H} \omega, \alpha_{H} d \omega \in \Omega^{q}(*(\mathcal{A} \backslash\{H\}))$. This implies that $\omega \in \Omega^{q}(\mathcal{A}, \mathcal{B})$.

To say something about $H^{*}\left(L_{0}, \mathcal{F}^{q}\right)$ we compare $\mathcal{F}^{q}$ with a coherent sheaf on an affine subset of $V$. Put

$$
V(\mathcal{A}, \mathcal{B})=V \backslash \bigcup_{H \in \mathcal{A} \backslash \mathcal{B}} H
$$

Clearly $V(\mathcal{A}, \mathcal{B})$ is an open affine subset of $V$ with the coordinate $\operatorname{ring} S(\mathcal{A}, \mathcal{B})=$ $S_{Q / Q(\mathcal{B})}$. The $S(\mathcal{A}, \mathcal{B})$-module $\Omega^{q}(\mathcal{A}, \mathcal{B})$ is finitely generated and therefore defines a coherent sheaf $\tilde{\Omega}^{q}(\mathcal{A}, \mathcal{B})$ on $V(\mathcal{A}, \mathcal{B})$. The following proposition shows the connection of this sheaf with $\mathcal{F}^{q}$.

\section{Proposition 3.2.}

$$
H^{p}\left(V(\mathcal{A}, \mathcal{B}) \backslash U, \tilde{\Omega}^{q}(\mathcal{A}, \mathcal{B})\right)=H^{p}\left(L_{0}, \mathcal{F}^{q}\right)
$$

for every $p$. 
Proof. For every atom $X$ of $L_{0}$ we consider the open subset $\bar{L}_{X}=\left\{Y \in L_{0} \mid Y \geq X\right\}$ of $L_{0}$ and the affine open subset

$$
V_{X}=V(\mathcal{A}, \mathcal{B}) \backslash \bigcup_{H \in \mathcal{B} \backslash \mathcal{A}_{X}} H
$$

of $V(\mathcal{A}, \mathcal{B})$. Clearly these sets define coverings of $L_{0}$ and $V(\mathcal{A}, \mathcal{B}) \backslash U$ respectively. The nerves of these coverings with the coefficient systems on them generated by $\mathcal{F}^{q}$ and $\tilde{\Omega}^{q}(\mathcal{A}, \mathcal{B})$ respectively can be naturally identified with one another. Besides any intersection of sets of either of the coverings is acyclic. In the case of the covering $\left\{\bar{L}_{X}\right\}$ it is true for any sheaf since any intersection has a unique minimal element. In the case of the covering $\left\{V_{X}\right\}$ it is true for any coherent sheaf since any intersection is affine. The result follows now from Corollary to Theorem 5.4.1 of [3].

Corollary 3.3. If $\mathcal{B} \neq \mathcal{A}$ then $H^{p}\left(L_{0}, \mathcal{F}^{q}\right)=0$ for every $p>0$ and every $q$.

Proof. If $\mathcal{B} \neq \mathcal{A}$ then $V(\mathcal{A}, \mathcal{B}) \backslash U=V(\mathcal{A}, \mathcal{B})$ that is affine. Now the result follows from Proposition 3.2.

Now we are ready to prove the main result of this section.

Proposition 3.4. If either $\mathcal{B} \neq \mathcal{A}$ or $\mathcal{A}$ is essential and tame then

$$
H^{p}\left(L_{0}, \mathcal{F}^{q}\right)=0 \text { for } 0<p \leq \ell-q-2 .
$$

Proof. If $\mathcal{B} \neq \mathcal{A}$ then the result follows from Corollary 3.3. Suppose $\mathcal{B}=\mathcal{A}$. Recall that $S(\mathcal{A}, \mathcal{A})=S, V(\mathcal{A}, \mathcal{A})=V$, and $\Omega^{*}(\mathcal{A}, \mathcal{A})=\Omega^{*}(\mathcal{A})$. Since $\mathcal{A}$ is tame $\operatorname{pd}_{S} \Omega^{q}(\mathcal{A}) \leq q$. Thus by the graded version of the Auslander-Buchsbaum formula $\operatorname{depth}_{S_{+}} \Omega^{q}(\mathcal{A}) \geq \ell-q$. Since $\mathcal{A}$ is essential $U=0$ and $I(U)=S_{+}$. Thus the above inequality implies that $H_{U}^{p}\left(V, \tilde{\Omega}^{q}(\mathcal{A})\right)=0$ for $p<\ell-q$ (e.g., see [5], Exercises 3.3.3 and 3.3.4). Now it follows from the exact sequence from [5], Exercise 3.2.3(e), that $H^{p}\left(V \backslash U, \tilde{\Omega}^{q}(\mathcal{A})\right)=0$ for $0<p \leq \ell-q-2$. Applying Proposition 3.2 we complete the proof.

\section{THE CASE OF $\operatorname{rk} \mathcal{A}=\operatorname{rk} \mathcal{B}$}

In this section we use the sheaves $\mathcal{F}^{q}$ to start proving Theorem 2.2 by two inductions. The outside induction is on $\mathrm{rk} \mathcal{B}$. We will prove the base of this induction together with the inductive step. So we consider an arbitrary pair $(\mathcal{A}, \mathcal{B})(\mathcal{B} \subset \mathcal{A})$ of arrangements and if $r=\operatorname{rk} \mathcal{B} \geq 1$ we assume that the result holds for every pair satisfying the condition of the theorem and with rank of the second arrangement smaller than $r$.

The inside induction is on the number of elements of $\mathcal{A}$ whose functionals are not linear combinations of the functionals of elements of $\mathcal{B}$. For the base of this induction we consider the case when $\operatorname{rk} \mathcal{A}=\operatorname{rk} \mathcal{B}=r$; i.e., the functional of every element of $\mathcal{A}$ is a linear combination of the functionals of elements of $\mathcal{B}$. Also using (T1) we can assume that $\mathcal{A}$ is essential, i.e., $r=\ell$. To handle this case we apply the sheaves $\mathcal{F}^{q}$.

If $\ell=0$ or 1 then the result is obvious. Suppose $\ell \geq 2$. Since $\operatorname{rk} \mathcal{A} \geq 2$ we have, due to Lemma 3.1, $\Gamma\left(L_{0}, \mathcal{F}^{q}\right)=\Omega^{q}(\mathcal{A}, \mathcal{B})$. The exterior differential $d$ defines the complex of sheaves

$$
0 \rightarrow \mathcal{F}^{0} \stackrel{d}{\rightarrow} \mathcal{F}^{1} \stackrel{d}{\rightarrow} \cdots \stackrel{d}{\rightarrow} \mathcal{F}^{\ell} \rightarrow 0
$$


We consider both spectral sequences generated by this complex (see [3], 2.4.5). One of them can be easily computed using the induction hypothesis of the outside induction. Indeed

$$
{ }^{\prime} E_{2}^{p q}=H^{p}\left(L_{0}, \mathcal{H}^{q}\right)
$$

where $\mathcal{H}^{q}$ is the $q$-th cohomlogy sheaf of the above complex. Since $\operatorname{rk} \mathcal{A}=\operatorname{rk} \mathcal{B}$ we have $\operatorname{rk}\left(\mathcal{A}_{X} \cap \mathcal{B}\right)<\ell=r$ for every $X \in L_{0}$. Besides since $\mathcal{A}_{X} \cap \mathcal{B} \subset \mathcal{B}$ every pair $\left(\mathcal{A}, \mathcal{A}_{X} \cap \mathcal{B}\right)$ satisfies the condition of the theorem. Thus by the induction hypothesis of the outside induction $\mathcal{H}^{q}$ is the locally constant sheaf with stalk $A_{q}(\mathcal{A})$. Since $L_{0}$ has the unique maximal element $V$, the sheaf $\mathcal{H}^{q}$ is acyclic on it whence ' $E_{2}^{0 q}=A_{q}(\mathcal{A})$ for every $q$ and all other terms ${ }^{\prime} E_{2}^{p q}$ vanish.

Now we study the second spectral sequence. We have

$$
{ }^{\prime \prime} E_{2}^{p q}=H^{p}\left(H^{q}\left(L_{0}, \mathcal{F}^{*}\right)\right)
$$

where the outside cohomologies are taken with respect to the differential $d$. Using again Lemma 3.1, we have " $E_{2}^{p 0}=H^{p}\left(\Omega^{*}(\mathcal{A}, \mathcal{B})\right)$. Besides if $\mathcal{B}=\mathcal{A}$ than the condition of the theorem implies that $\mathcal{A}$ is tame. Hence due to Proposition 3.4, $" E_{2}^{p q}=0$ for $0<q \leq \ell-p-2$. Thus only differentials able to kill elements of " $E_{s}^{p 0}$ for some $s$ are $d_{q+1}:{ }^{\prime \prime} E_{q}^{\ell-q-1, q} \rightarrow{ }^{\prime \prime} E_{q+1}^{\ell, 0}, q=1,2, \ldots$ Comparing the $E_{\infty}$ terms of the two spectral sequences we obtain the following lemma.

Lemma 4.1. For every $p \neq \ell$ the canonical homomorphism $H^{p}\left(\Omega^{*}(\mathcal{A}, \mathcal{B})\right) \rightarrow$ $A_{p}(\mathcal{A})$ of the spectral sequence " $E_{s}^{p q}$ is an isomorphism.

To handle the top cohomology it suffices to compare the Euler characteristics (cf. also Proposition 6.1).

It is well known that $\chi(A(\mathcal{A}))=0$ for any central arrangement $\mathcal{A}$. In order to compute the other Euler characteristic we apply the techniques from Section 3 of [13]. As usual we suppose that the hyperplane $\left\{x_{1}=0\right\}$ is in $\mathcal{A}$. For every $p$ there are two $S$-module homomorphisms

$$
\phi: \Omega^{p}(\mathcal{A}, \mathcal{B}) \rightarrow \Omega^{p-1}(\mathcal{A}, \mathcal{B}) \text { and } \delta: \Omega^{p}(\mathcal{A}, \mathcal{B}) \rightarrow \Omega^{p+1}(\mathcal{A}, \mathcal{B})
$$

via $\phi(\omega)=\left\langle\theta_{E}, \omega\right\rangle$ and $\delta(\omega)=\frac{d x_{1}}{x_{1}} \wedge \omega$ where

$$
\theta_{E}=\sum_{i=1}^{\ell} x_{i} \partial / \partial x_{i}
$$

is the Euler derivation and $\langle$,$\rangle is the inner product.$

The following equalities are very well known and can be checked easily

$$
\begin{gathered}
\phi^{2}=0, \delta^{2}=0, \\
\phi \delta+\delta \phi=\mathrm{id}_{\Omega^{p}}, \\
\delta d=-d \delta, \\
\phi d(\omega)+d \phi(\omega)=\operatorname{tdeg} \omega
\end{gathered}
$$

where $\omega$ is homogeneous of degree $g$ from $\Omega^{p}(\mathcal{A}, \mathcal{B})$ and $\operatorname{tdeg} \omega=p+g$ (the total degree of $\omega)$.

Since all the maps $d, \delta$, and $\phi$ preserve the total degree, (4) implies that the subcomplex $\Omega_{0}^{*}$ of $\Omega^{*}(\mathcal{A}, \mathcal{B})$ of 0 total degree is quasi-isomorphic to the whole complex. The equalities (1)-(4) imply that this complex splits into direct sum of its two subcomplexes

$$
\Omega_{0}^{*}=K_{0}^{*} \oplus K_{1}^{*}
$$


where $K_{0}^{*}=\operatorname{ker} \phi$ and $K_{1}^{*}=\operatorname{ker} \delta$. Besides the map $\delta: K_{0}^{*} \rightarrow K_{1}^{*}$ skew commutes with $d$ whence it is a quasi-isomorphism of degree +1 . In particular

$$
H^{\ell-1}\left(\Omega_{0}^{*}\right) \cong H^{\ell-1}\left(K_{1}^{*}\right) \oplus H^{\ell}\left(K_{1}^{*}\right)
$$

and

$$
H^{\ell}\left(\Omega_{0}^{*}\right)=H^{\ell}\left(K_{1}^{*}\right)
$$

Since Lemma 4.1 implies that $\operatorname{dim}_{F}\left(H^{\ell-1}\left(\Omega_{0}^{*}\right)\right)<\infty$ we have

$$
\operatorname{dim}_{F}\left(H^{\ell}\left(\Omega_{0}^{*}\right)\right)<\infty
$$

and

$$
\sum_{i=0}^{\ell}(-1)^{i} \operatorname{dim}_{F}\left(H^{i}\left(\Omega_{0}^{*}\right)\right)=0,
$$

which completes the proof for the case $\operatorname{rk} \mathcal{A}=\operatorname{rk} \mathcal{B}$ (the base of the inside induction).

\section{The General CASE}

In this section we consider an arbitrary pair $(\mathcal{A}, \mathcal{B})(\mathcal{B} \subset \mathcal{A})$ satisfying the condition of the theorem. Again without loss of generality we can assume that $\mathcal{A}$ is essential, i.e., $\operatorname{rk} \mathcal{A}=\ell$. Denote by $W$ the subspace of $V^{*}$ generated by the functionals of all the hyperplanes of $\mathcal{B}$ and put $\overline{\mathcal{B}}=\mathcal{A} \cap W$. Also enumerate the elements of $\mathcal{A} \backslash \overline{\mathcal{B}}$, so $\mathcal{A} \backslash \overline{\mathcal{B}}=\left\{H_{1}, \ldots, H_{k}\right\}$. Clearly $\Omega^{*}(\mathcal{A}, \mathcal{B})=\Omega^{*}(\overline{\mathcal{B}}, \mathcal{B})_{Q / Q(\overline{\mathcal{B}})}$.

First we need to prove a lemma.

Lemma 5.1. Fix a basis $\left(x_{1}, \ldots, x_{\ell}\right)$ of $V^{*}$ and suppose that $x_{1} \notin W$. Suppose that $\omega \in \Omega^{*}(\mathcal{A}, \mathcal{B})$ and $\omega=d x_{1} \wedge \omega_{1}+\omega_{2}$ where $\omega_{i}$ do not contain $d x_{1}$ in their standard basis representations. Then $\omega_{i} \in \Omega^{*}(\mathcal{A}, \mathcal{B})$.

Proof. Represent $\omega=\omega_{0} / \pi$ where $\omega_{0} \in \Omega^{*}(\mathcal{B})$ and $\pi$ is the product of the linear functionals of elements from $\mathcal{A} \backslash \mathcal{B}$ with some multiplicities. Then the condition on $\omega$ means that $\omega_{0}=d x_{1} \wedge \omega_{01}+\omega_{02}$ and $\omega_{i}=\omega_{0 i} / \pi$. Since $x_{1}$ is generic with respect to $\mathcal{B}$ it is clear that $\omega_{0 i} \in \Omega^{*}(\mathcal{B})$, which concludes the proof.

Now we are ready to prove that $H^{*}(\Omega(\mathcal{A}, \mathcal{B}))=A(\mathcal{A})$ by induction on $k$. For $k=$ 0 we have $\mathcal{A}=\overline{\mathcal{B}}$ and as it is proved in the previous section $H^{*}(\Omega(\overline{\mathcal{B}}, \mathcal{B}))=A(\overline{\mathcal{B}})$. Suppose $k \geq 1$ and change the basis in $V^{*}$ so that $\beta_{k}=x_{1}$. Put $\Omega^{*}=\Omega^{*}(\mathcal{A}, \mathcal{B})$, $\mathcal{A}^{\prime}=\mathcal{A} \backslash\left\{H_{k}\right\}$, and $\Omega_{(0)}^{*}=\Omega^{*}\left(\mathcal{A}^{\prime}, \mathcal{B}\right)$. Clearly $\Omega^{*}=\left(\Omega_{(0)}^{*}\right)_{x_{1}}$. Consider the following decreasing filtration of the complex $\Omega^{*}$ :

$$
\Omega_{p}^{p+q}=\left\{\omega \in \Omega^{p+q} \mid x_{1}^{q} \omega \in \Omega_{(0)}^{*}\right\}
$$

where $q \geq 0,-\infty<p \leq \ell$ and $-p \leq q \leq \ell-p$ (all other terms are 0 ).

Our goal is to compute $H^{*}\left(\Omega^{*}\right)$ using the spectral sequence of the above filtration. Recall that $E_{1}^{p q}=H^{p+q}\left(E^{p *}\right)$ where $E^{p *}=\Omega_{p}^{*} / \Omega_{p+1}^{*}$. The differential $d_{0}$ in the latter complex is induced by $d$ and a simple calculation shows that $d_{0}=-q d x_{1} / x_{1} \wedge$ for every $q \geq 0$. Now we observe

(i) For every $p<0$ the complex $E^{p *}$ is acyclic.

Indeed suppose that $\omega \in \Omega_{p}^{p+q}$ and $d x_{1} / x_{1} \wedge \omega \in \Omega_{p+1}^{p+q+1}$. This implies that $\omega=d x_{1} \wedge \omega_{1}+x_{1} \omega_{2}$ where $\omega_{i}$ does not have $d x_{1}$ in it whence due to Lemma 5.1 $\omega_{i} \in \Omega^{*}$. This implies that to represent the cocycle from $E^{p, p+q}$ one can choose $\omega=d x_{1} \wedge \omega_{1}$. This gives $\omega=d x_{1} / x_{1} \wedge x_{1} \omega_{1}$ and proves the claim since $q \neq 0$ in this case. 
(ii) Let $0 \leq p \leq \ell$. Then $E_{1}^{p 0}=\Omega_{(0)}^{p}$,

$$
E_{1}^{p 1}=\bar{\Omega}^{p}=\left(d x_{1} / x_{1} \wedge \Omega_{(0)}^{p}\right) /\left(\Omega_{(0)}^{p+1} \cap\left(d x_{1} / x_{1} \wedge \Omega_{(0)}^{p}\right)\right),
$$

and $E_{1}^{p q}=0$ otherwise.

Indeed the same reasoning as in the proof of (i) shows that only dimensions where the complex $E^{p *}$ can be non-acyclic are $p$ and $p+1$. In dimension $p$ the computation of cohomology is trivial; in dimension $p+1$, it can be done in the same way as in (i).

Claims (i) and (ii) give the whole picture of $E_{1}^{p q}$. It has 2 nonvanishing rows for $q=0$ and $q=1$. Computing the differentials of $E_{1}$ in the standard way we see that the row for $q=0$ forms the complex $\left(\Omega_{(0)}^{*}, d\right)$ and the row for $q=1$ forms the complex $\bar{\Omega}^{*}$ with the differential induced by $d$. Clearly the pair $\left(\mathcal{A}^{\prime}, \mathcal{B}\right)$ still satisfies the condition of the theorem. Thus by the inductive hypothesis of the inside induction (on $k$ ) we have $E_{2}^{p 0}=H^{p}\left(\Omega_{(0)}^{*}\right)=A_{p}\left(\mathcal{A} \backslash\left\{H_{k}\right\}\right)$.

To finish the calculation of $E_{2}$ we need to find $E_{2}^{p 1}$. For that denote by $\mathcal{A}^{\prime \prime}$, $\mathcal{B}^{\prime \prime}$, and $(\mathcal{A} \backslash \mathcal{B})^{\prime \prime}$ the restrictions of $\mathcal{A}, \mathcal{B}$, and $\mathcal{A} \backslash \mathcal{B}$ respectively to $H_{k}$. Notice that the arrangements $\mathcal{B}^{\prime \prime}$ and $(\mathcal{A} \backslash \mathcal{B})^{\prime \prime}$ may have a non-empty intersection. Put $\mathcal{B}_{0}=\mathcal{B}^{\prime \prime} \backslash(\mathcal{A} \backslash \mathcal{B})^{\prime \prime}$.

Lemma 5.2. The complex $\bar{\Omega}^{*}$ is isomorphic to the complex $\Omega^{*}\left(\mathcal{A}^{\prime \prime}, \mathcal{B}_{0}\right)$.

Proof. For each $\omega \in \Omega_{(0)}^{*}$ represent $\omega=d x_{1} \wedge \omega_{1}+\omega_{2}$ where $\omega_{i}$ does not have $d x_{1}$ in it and put $\phi(\omega)=\left.\omega_{2}\right|_{x_{1}=0}$. The following claims are straightforward and together they prove the statement.

(i) $\phi(\omega) \in \Omega^{*}\left(\mathcal{A}^{\prime \prime}, \mathcal{B}_{0}\right)$.

(ii) The formula $\psi\left(d x_{1} / x_{1} \wedge \omega\right)=\phi(\omega)$ defines a linear map $\psi: d x_{1} / x_{1} \wedge \Omega_{(0)}^{*} \rightarrow$ $\Omega^{*}\left(\mathcal{A}^{\prime \prime}, \mathcal{B}_{0}\right)$.

(iii) The map $\psi$ commutes with the exterior differential.

(iv) The map $\psi$ is surjective and its kernel is $\left(d x_{1} / x_{1} \wedge \Omega_{(0)}^{*}\right) \cap \Omega_{(0)}^{*}$.

Now we are able to finish the computation of $E_{2}$. By Lemma $5.2, E_{2}^{p 1}=$ $H^{p}\left(\Omega^{*}\left(\mathcal{A}^{\prime \prime}, \mathcal{B}_{0}\right)\right)$. Clearly either $\operatorname{rk} \mathcal{B}_{0}<r$ or the number of elements of $\mathcal{A}^{\prime \prime}$ whose functionals are not linear combinations of functionals of elements of $\mathcal{B}_{0}$ is less than $k$. Let us check that the pair $\left(\mathcal{A}^{\prime \prime}, \mathcal{B}_{0}\right)$ satisfies the condition of the theorem. Suppose that $\mathcal{B}_{1} \subset \mathcal{B}_{0}$ and $B_{1}$ is linearly closed in $\mathcal{A}^{\prime \prime}$. Since linear projection defined by evaluation at $x_{1}=0$ is injective on $W, \mathcal{B}_{1}$ is isomorphic to a subarrangement of $\mathcal{B}$ linearly closed in $\mathcal{A}$. By the condition of the theorem $\mathcal{B}_{1}$ is tame. Hence using the inductive hypothesis of either the outside or the inside induction we have $E_{2}^{p 1}=A^{p}\left(\mathcal{A}^{\prime \prime}\right)$. Besides $E_{2}^{p q}=0$ for $q>1$.

Now it follows from the exact sequence from Theorem 3.65 of [7] that all elements of non-zero terms of $E_{2}$ can be represented by distinct elements of $A(\mathcal{A})$, in particular by closed forms. Thus all this elements survive in $E_{\infty}$ and the natural embedding $A(\mathcal{A}) \subset \Omega^{*}(\mathcal{A}, \mathcal{B})$ is a quasi isomorphism. This concludes the proof.

Remark 5.3. Analyzing the proof (of Lemma 4.1 in particular) one sees that the tame condition of Theorem 2.2 has been used only for third cohomology and higher. Thus one obtains that

$$
H^{p}\left(\Omega^{*}(\mathcal{A}, \mathcal{B})\right)=A_{p}(\mathcal{A})
$$

for $0 \leq p \leq 2$ and every pair $(\mathcal{A}, \mathcal{B})$. 


\section{The Highest COHOMOLOGY}

In this section we show that the conjecture from Introduction is true for the highest cohomology and every central arrangement $\mathcal{A}$. This implies that Corollary 2.3 holds for every central arrangement in dimension 4 .

Proposition 6.1. For every central arrangement $\mathcal{A}$

$$
H^{\mathrm{rk} \mathcal{A}}\left(\Omega^{*}(\mathcal{A})=A_{\mathrm{rk} \mathcal{A}}(\mathcal{A}) .\right.
$$

Proof. As usual we can assume that $\mathcal{A}$ is essential, i.e., $\operatorname{rk} \mathcal{A}=\ell$. We use the idea developed in [15] and introduce specific forms from $\Omega^{*}(\mathcal{A})$. If $X \in L$ then we can choose a maximal system $\beta_{1}, \ldots, \beta_{k}$ of linearly independent elements from $\mathcal{A}_{X}\left(k=\operatorname{rk} \mathcal{A}_{X}\right)$ and put $d v_{X}=d \beta_{1} \wedge \cdots \wedge d \beta_{k}$ and $\omega_{X}=d v_{X} / Q_{X}$ where $Q_{X}=$ $\prod_{H \in \mathcal{A}_{X}} \alpha_{H}$. Clearly $\omega_{X}$ is defined by $X$ up to a non-zero multiplicative constant, $\omega_{X} \in \Omega^{k}(\mathcal{A})$, and $d \omega_{X}=0$. Moreover $\omega_{0}$ freely generates $\Omega^{\ell}(\mathcal{A})$ over $S$.

Recall from the last part of Section 4 that $H^{*}\left(\Omega^{*}(\mathcal{A})\right)=H^{*}\left(\Omega_{0}^{*}\right)$ where $\Omega_{0}^{*}$ is the complex of logarithmic forms of total degree 0 . By above we have $\Omega_{0}^{\ell} \cong S_{n-\ell}$ $(n=|\mathcal{A}|)$ whence $\operatorname{dim} \Omega_{0}^{\ell}=\left(\begin{array}{c}n-1 \\ \ell-1\end{array}\right)$. Now we put $L_{1}=\left\{X \in L \mid \operatorname{dim}_{F} X=1\right\}$ and compute $d B \subset \Omega_{0}^{\ell}$ where $B=\left(\sum_{X \in L_{1}} S \omega_{X}\right) \cap \Omega_{0}^{\ell-1}$. For that fix $X \in L_{1}$ and change the basis in $V^{*}$ so that $x_{1}, \ldots, x_{\ell-1} \in \mathcal{A}_{X}$ and $x_{\ell} \in \mathcal{A} \backslash \mathcal{A}_{X}$. Then for any $f \in S$ we have

$$
d\left(f \omega_{X}\right)=\frac{\partial f}{\partial x_{\ell}} d x_{\ell} \wedge \omega_{X}=c \frac{\partial f}{\partial x_{\ell}} \pi_{X} \omega_{0}
$$

where $c \in F \backslash\{0\}$ and $\pi_{X}=Q / Q_{X}$. This implies that $d B$ is linearly isomorphic to the homogeneous component $I_{n-\ell}$ of degree $n-\ell$ of the ideal $I=\sum_{X \in L_{1}} S \pi_{X}$ of $S$. The Hilbert series of $I$ has been computed in [15]. To recall this denote by $d_{j}(i)$ the number of subsets $T$ of $L_{1}$ such that $|T|=j+1$ and $\left|\bigcap_{X \in T} \mathcal{A}_{X}\right|=k$. Then put $\chi_{i}=\sum_{j \geq 0}(-1)^{j} d_{j}(i)$. Now

$$
\operatorname{Poin}(I, t)=(1-t)^{\ell} \sum_{i=0}^{n-1} \chi_{i} t^{n-i}
$$

is the Hilbert series of $I$. This implies

$$
\operatorname{dim}_{F} I_{n-\ell}=\sum_{i \geq \ell} \chi_{i}\left(\begin{array}{l}
i-1 \\
\ell-1
\end{array}\right) .
$$

Now we need a combinatorial lemma.

Lemma 6.2. In the above notation

$$
(-1)^{\ell} \mu(L)=\left(\begin{array}{c}
n-1 \\
\ell-1
\end{array}\right)-\sum_{i \geq \ell} \chi_{i}\left(\begin{array}{c}
i-1 \\
\ell-1
\end{array}\right)
$$

where $\mu(L)$ is the value $\mu(0, V)$ of the Möbius function $\mu$ of $L$.

Proof. As it is well known $\mu(L)=\mu\left(L^{\mathrm{op}}\right)$. Due to Lemma 2.35 of [7], $\mu\left(L^{\mathrm{op}}\right)=$ $\sum(-1)^{|\mathcal{B}|}$ where $\mathcal{B}$ is running through all the essential subarrangements of $\mathcal{A}$. Denote by $\sigma_{i}$ the contribution to this sum of the subarrangements of $i$ elements $(i \geq \ell)$. Then using the inclusion-exclusion formula we have 


$$
\sigma_{i}=\left(\begin{array}{c}
n \\
i
\end{array}\right)-\sum_{j \geq i} \chi_{j}\left(\begin{array}{l}
j \\
i
\end{array}\right)
$$

whence

$$
\mu(L)=\sum_{i \geq \ell}(-1)^{i}\left(\left(\begin{array}{c}
n \\
i
\end{array}\right)-\sum_{j \geq i} \chi_{j}\left(\begin{array}{l}
j \\
i
\end{array}\right)\right) .
$$

The result follows now from standard properties of binomial coefficients.

Now we are able to finish the computation of $H^{\ell}\left(\Omega^{*}\right)$. We know that $A_{\ell} \subset \Omega_{0}^{\ell}$ and $\operatorname{dim}_{F} A_{\ell}=(-1)^{\ell} \mu(L)$. Besides by Corollary $2.5, d \Omega^{\ell-1} \cap A_{\ell}=0$. On the other hand

$$
\operatorname{dim} \Omega_{0}^{\ell}=\left(\begin{array}{l}
n-1 \\
\ell-1
\end{array}\right)=(-1)^{\ell} \mu(L)+\sum_{i \geq \ell} \chi_{i}\left(\begin{array}{l}
i-1 \\
\ell-1
\end{array}\right)=\operatorname{dim} A_{\ell}+\operatorname{dim} d B .
$$

Thus $A_{\ell} \cong H^{\ell}\left(\Omega^{*}\right)$, which completes the proof of the proposition.

Corollary 6.3. Corollary 2.3 holds for every central arrangement in dimension 4.

Proof. Due to Remark $5.3 H^{i}\left(\Omega^{*}\right)=A_{i}(\mathcal{A})$ for $i=0,1$ and 2. Proposition 6.1 implies that it is also true for $i=4$. Now the result follows by comparing the Euler characteristic similarly to the last part of Section 4.

\section{REFERENCES}

1. Brieskorn, E.: Sur les groupes de tresses, In: Séminaire Bourbaki 1971/1972, Lecture Notes in Math. 317 (1973), 21-44. MR 54:10660

2. Castro, F., Narváez, L., and Mond, D.: Cohomology of the complement of a free divisor, preprint, 1994.

3. Godement, R.: Topologie algébrique et théorie des faisceaux. Hermann, Paris, 1958. MR 21:1583

4. Grothendieck, A.: On the de Rham cohomology of algebraic varieties, Publ. Math. de l'I.H.E.S. 29 (1966), 95-103. MR 33:7343

5. Hartshorne, R.: Algebraic Geometry. Springer Verlag, 1977. MR 57:3116

6. Orlik, P., Solomon, L.: Combinatorics and topology of complements of hyperplanes, Invent. Math. 56 (1980), 167-189. MR 81e:32015

7. Orlik, P., Terao, H.: Arrangements of Hyperplanes. Grundlehren der Math. Wiss. 300, Springer Verlag, 1992. MR 94e:52014

8. Orlik, P., Terao, H.: Arrangements and Milnor fibers, preprint, 1993.

9. Rose, L., Terao, H.: A free resolution of the module of logarithmic forms of a generic arrangement, J. of Algebra 136 (1991), 376-400. MR 93h:32048

10. Solomon, L., Terao, H.,: A formula for the characteristic polynomial of an arrangement, Adv. Math. 64 (1987), 305-325. MR 88m:32022

11. Terao, H.,: Forms with logarithmic pole and the filtration by the order of the pole, Proc. Internat. Sympos. on Algebraic Geometry, Kyoto, 1977, Kinokuniya, Tokyo, 1978, 673-685. MR 82b:14004

12. Stückrad, J., Vogel, W.: Buchsbaum Rings and Applications, Springer-Verlag, 1986. MR 88h:13011b

13. Terao, H., Yuzvinsky, S.: Logarithmic forms on affine arrangements, preprint, 1994.

14. Yuzvinsky, S.: Cohomology of local sheaves on arrangement lattices, Proceedings of AMS 112 (1991), 1207-1217. MR 91j:52016

15. Yuzvinsky, S.: Generators of the module of logarithmic 1-forms with poles along an arrangement, J. Algebra Combinatorics 4 (1995), 253-269. MR 96b:32044

Department of Mathematical Sciences, University of Alaska, Fairbanks, Alaska 99775

Department of Mathematics, University of Oregon, Eugene, Oregon 97403 